\title{
Hidradenitis suppurativa: an update
}

\author{
Author: John R Ingram ${ }^{A}$
}

Hidradenitis suppurativa (HS) is a chronic, painful skin disease characterised by recurrent inflammatory lesions in flexural locations such as the axillae, groins and perineum. The papules, nodules and abscesses may discharge bloodstained pus which, combined with pain, results in marked quality-of-life reduction. Sinus tracts and scarring may also result. Onset of HS is typically in the second to fourth decades and it affects about $1 \%$ of young European adults. There are links with smoking and obesity and an autosomal dominant pattern of inheritance is reported by one-third of patients. Medical management escalates from topical antimicrobials to oral tetracyclines, a combination of clindamycin and rifampicin typically given for 10 weeks, oral disease modifiers, and anti-tumour necrosis factor-alpha therapies. Excision of individual lesions has high recurrence rates which can be minimised by wider excisions, at the expense of longer healing times. Treatment of pain is a relatively neglected aspect of therapy.

\section{Clinical presentation}

Hidradenitis suppurativa (HS), also known as acne inversa, is a chronic skin disease characterised by recurrent inflammatory lesions in flexural sites, typically the axillae, groins, perineum and buttocks, and also the inframammary region in women. Lesions may also be present in non-flexural sites such as the back. In the absence of pathognomonic tests, a consensus disease definition requires a history of at least five typical HS lesions, namely erythematous papules, nodules or abscesses, in flexural sites. ${ }^{1}$ Acute lesions are usually painful and more superficial lesions may discharge blood-stained pus, producing odour and staining clothing. Lesions tend to leave permanent scars, often rope-like in appearance, and subcutaneous sinuses may form (Fig 1). In severe HS, lesions, scars and sinuses coalesce into chronic inflammatory, suppurative skin plaques. Not surprisingly, HS can have a large impact on quality of life, equivalent to severe psoriasis, ${ }^{2}$ and results in functional impairment similar or greater than heart disease, diabetes or asthma, when measured by the EQ5D scale. ${ }^{3}$

Author: ${ }^{\text {A }}$ senior lecturer and consultant dermatologist, Department of Dermatology and Wound Healing, Division of Infection and Immunity, Cardiff University, Cardiff, UK

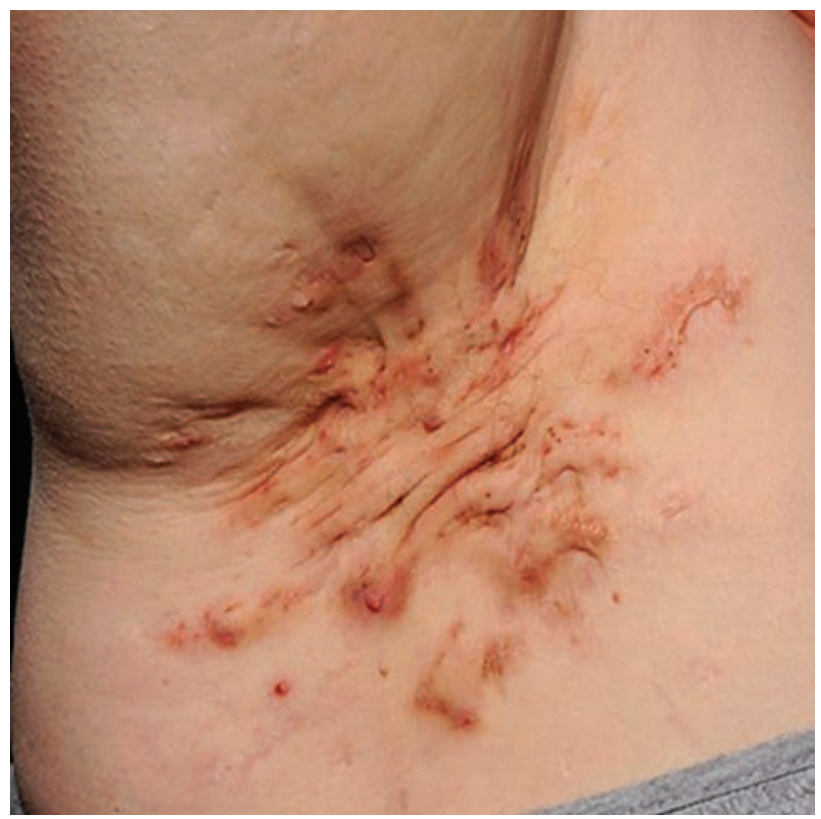

Fig 1. Characteristic rope-like scars and sinus formation in the axilla of a person with hidradenitis suppurativa.

\section{Epidemiology and disease severity scales}

Currently, the most robust data regarding HS incidence are derived from self-reported questionnaire studies, giving estimates of about $1 \%$ of the adult European population, with a female to male ratio of approximately $3: 1{ }^{4}$ Incidence is usually after puberty, between the second and fourth decades, which increases impact on work productivity and relationships. There is a strong association between HS and smoking, with a majority of patients being current or ex-smokers, as well as obesity. ${ }^{2}$ Data are limited regarding the effects of smoking cessation and weight loss on disease severity, however non-smokers are more likely to spontaneously remit. ${ }^{5}$ There is evidence that HS is associated with other chronic inflammatory conditions, in particular inflammatory bowel disease. ${ }^{6}$

The Hurley staging system is useful to define mild, moderate and severe disease (Fig 2). Clinical trials tend to use other physician-reported measures which involve lesion counts, such as the modified hidradenitis suppurativa score ${ }^{2}$ or 


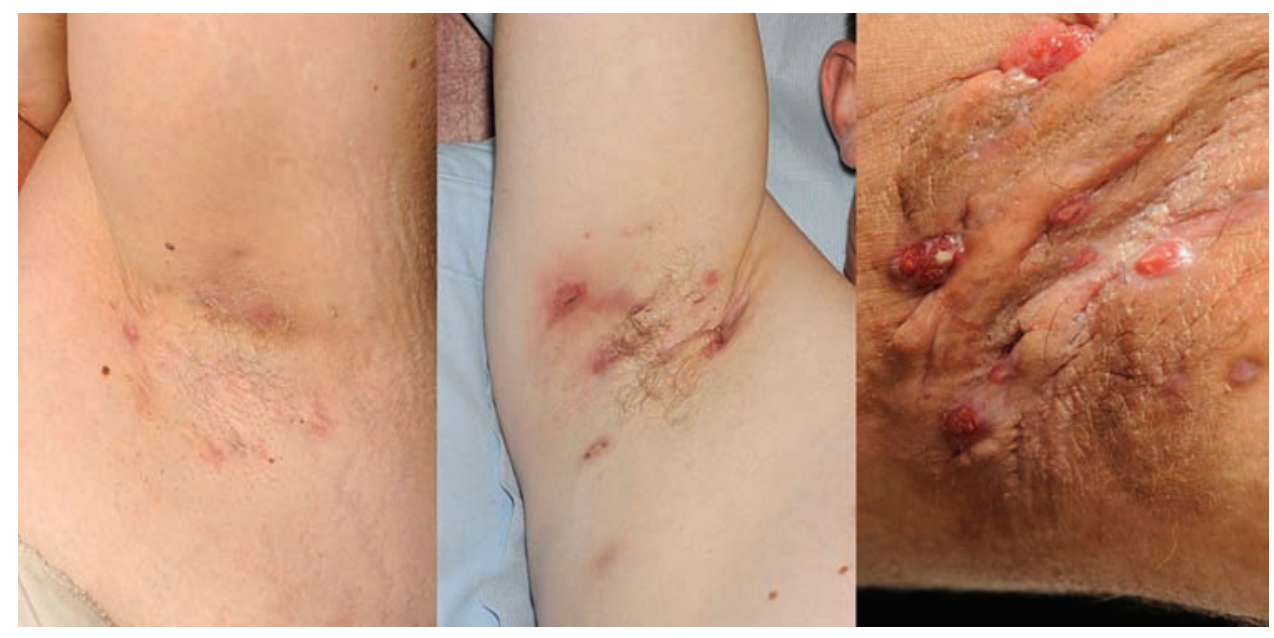

Fig 2. The Hurley staging system. (a) Stage 1 (mild) represents isolated lesions with minimal scarring; (b) stage 2 (moderate) is defined as multiple scarring lesions interspersed with normal skin; and (c) stage 3 (severe) represents coalesced lesions forming inflammatory plaques.
$\mathrm{HiSCR}^{7}$, as the Hurley system is relatively static. Patientreported outcomes include quality-of-life measures, such as the dermatology life quality index (DLQI ${ }^{8}$ and the pain visual analogue scale (VAS).

\section{Aetiology}

In most cases, the aetiology of HS is unknown and research is pursuing four main hypotheses: genetics, environmental factors, endocrine causes and microbiology. Approximately one-third of HS patients report a family history of HS, with an inheritance pattern consistent with autosomal dominant inheritance. ${ }^{1}$ Linkage studies in large Han Chinese families

\section{Key points}

HS is a chronic, painful skin condition involving multiple papules, nodules and abscesses in flexural sites, such as the axillae, groins and perineum, as well as non-flexural sites such as the back in some cases.

It affects approximately $1 \%$ of the adult European population and can have a large impact on quality of life, due to pain, discharge of pus and restriction of movement.

There is a link with smoking and obesity, although HS may affect non-smokers of normal weight.

There are multiple medical treatment options, including antibiotics, retinoids, immunomodulators and anti-TNF- $\alpha$ therapies, and surgical excision of affected regions may also be required; however the evidence base is limited.

Management of pain due to acute inflammatory lesions is a relatively neglected element of treatment.

KEYWORDS: Hidradenitis suppurativa, pain, quality of life, gamma secretase with severe HS also involving non-flexural sites demonstrated pathogenic mutations in several gamma-secretase genes ${ }^{9}$ but these mutations underpin only a small minority of European familial HS cases. ${ }^{10}$ Environmental factors include obesity, which may worsen or possibly trigger HS due to the frictional effects of larger skin folds, and smoking; there is no highquality trial evidence to support dietary influences currently. A possible endocrine cause is suggested by onset soon after puberty and a reported reduction in HS disease activity in around half of women after the menopause. ${ }^{5}$ The microbiology of HS is complicated by sinus tracts and it is difficult to determine if bacteria are commensal or pathogenic. The pathophysiology of HS is also incompletely understood, but histologically, hyperkeratosis of the opening of hair follicles is an early event, which may cause accumulation of apocrine gland secretions, rupture of the hair follicle and a subsequent chronic foreign body reaction to the exposed hair shaft. ${ }^{11}$

\section{Management}

UK guidelines for HS management are being developed currently but European guidelines are available. ${ }^{12}$ In the UK, the HS Trust offers an information and support service (www. hstrust.org) and the British Association of Dermatologists has a helpful patient leaflet (www.bad.org.uk/leaflets). Lifestyle modification, in terms of smoking cessation and weight loss, should be recommended where relevant, bearing in mind that HS can affect non-smokers of normal weight. There is a relative lack of high-quality evidence for HS therapy, with only 12 randomised controlled trials (RCTs) available, with an average of 27 participants.

Topical antimicrobials are given for mild disease, either topical antiseptics, such as chlorhexidine lotion for the shower, or topical antibiotics, such as clindamycin $1 \%$ solution. A recent survey of UK dermatologists found that oral tetracyclines are used as first-line oral therapy by $75 \% .^{13}$ The second-line therapy most commonly employed is a 10week course of clindamycin and rifampicin, both at a dosage of $300 \mathrm{mg}$ twice daily, based on case series evidence. ${ }^{14}$ Drug interactions with rifampicin should be considered and patients 
should be warned of a risk of gastrointestinal adverse effects, although excessive cases of Clostridium difficile diarrhoea have not been reported to date.

For moderate to severe disease, acitretin can be considered for males and non-fertile females, in the context that it has teratogenic potential for three years after treatment cessation. The mode of action may be to reduce hyperkeratosis of the hair follicle opening. Isotretinoin, a retinoid commonly used for severe acne, does not appear to be effective for most cases of HS. Oral immunomodulators most commonly used in the UK to control the inflammation are dapsone and cyclosporin, ${ }^{13}$ based on evidence from case series.

Several RCTs have been performed to assess the efficacy of anti-tumour necrosis factor-alpha (TNF $\alpha$ ) therapies for moderate to severe HS. Etanercept $50 \mathrm{mg}$ twice weekly did not show benefit while infliximab $5 \mathrm{mg} / \mathrm{kg}$ given at weeks 0,2 and 4 was effective compared with placebo at 12 weeks, in a relatively small RCT of 38 participants. ${ }^{15}$ In a larger three-arm trial of 154 participants comparing adalimumab $40 \mathrm{mg}$ weekly or adalimumab $40 \mathrm{mg}$ alternate weeks with placebo, there was no benefit with alternate week dosing. ${ }^{16}$ However, weekly dosing was effective, producing a 4-point benefit on the DLQI scale, matching the minimal clinically important difference for the outcome measure.

There are no RCTs to guide the timing or choice of procedure in HS. Incision and drainage may be performed to treat an isolated acute HS nodule but recurrence rates are high. For mild to moderate disease some European centres are now performing de-roofing of lesions, in which electrosurgery is used to lay open sinus tracts, rather than narrow margin excision with primary closure. ${ }^{17}$ For more extensive disease, wider excision is necessary, removing all of the apocrine glandbearing tissue of the region. The closure options are with skin flaps, skin grafting or secondary intention healing. There is a trade-off in choice of procedure between risk of recurrence and post-operative downtime. Case series evidence suggests that the lowest risk of recurrence is achieved by wider excision and secondary intention healing over several weeks, requiring a wound care support service. There has been one surgical RCT performed in HS, which investigated insertion of a gentamicin sponge as a post-operative adjunct following excision and primary closure; however overall this did not reduce postoperative complications relative to the placebo group. ${ }^{18}$

Laser and light treatments have been investigated in a few small RCTs, typically using a within-participant design, treating only one side of a bilaterally affected region. Interventions have included the carbon dioxide laser, which ablates skin in a similar way to excisional surgery, hair removal lasers, intense pulsed light (IPL) and photodynamic therapy. However most of the trials did not employ a sham placebo intervention and so, combined with their small number of participants, the quality of the evidence remains relatively low. In addition, many dermatology units do not have access to these interventions, limiting their widespread use.

\section{The future}

A recent priority setting partnership (PSP), involving a partnership between HS patients and clinicians, was asked to identify and prioritise HS research uncertainties. ${ }^{19}$ The PSP identified multiple therapy areas in which RCT data were lacking, as described above. In addition, management of an acute flare was given second highest priority and the related issue of pain management was also included in the top ten. At the moment, patients often receive multiple one-week courses of oral antibiotics to treat flares, when this may not alter the natural history of individual lesions. ${ }^{20}$ Alternatively, patients have to attend emergency departments to undergo incision and drainage of lesions. There is a need to ensure more joined-up care for HS patients, identifying the condition earlier and providing better long-term disease control. Optimal HS management in secondary care should ideally be based on the multidisciplinary team model, including expertise in dermatology, surgery, wound care, clinical psychology and pain management.

In summary, HS is a relatively common, painful, disabling condition which previously has been relatively neglected by the medical community and society in general, in part because it is embarrassing and hidden from view. Recent European guidelines recommend an escalating sequence of medical therapies, including topical antiseptics, oral tetracyclines and oral disease modifiers, followed by anti-TNF $\alpha$ therapies for severe disease, in parallel with local or wider surgical procedures. ${ }^{12}$ However many important questions remain, due to a relative lack of high-quality clinical trials.

\section{Conflicts of interest}

JRI is Cardiff PI for an observational HS study sponsored by Abbvie. He holds a Health and Care Research Wales (HCRW) Health Fellowship. The views expressed in this publication are those of the author and not necessarily those of HCRW.

\section{References}

1 von der Werth JM, Williams HC, Raeburn JA. The clinical genetics of hidradenitis suppurativa revisited. Br J Dermatol 2000;142:94753.

2 Sartorius K, Emtestam L, Jemec GB, Lapins J. Objective scoring of hidradenitis suppurativa reflecting the role of tobacco smoking and obesity. Br J Dermatol 2009;161:831-9.

3 Mikkelsen PR, Vinding GR, Ring HC, Jemec GB. Disutility in patients with hidradenitis suppurativa: a cross-sectional study using EuroQoL-5D. Acta Derm Venereol 2015, epub ahead of print.

4 Revuz JE, Canoui-Poitrine F, Wolkenstein P et al. Prevalence and factors associated with hidradenitis suppurativa: results from two case-control studies. J Am Acad Dermatol 2008;59:596-601.

5 Kromann CB, Deckers IE, Esmann S et al. Risk factors, clinical course and long-term prognosis in hidradenitis suppurativa: a cross-sectional study. Br J Dermatol 2014;171:819-24.

6 van der Zee HH, van der Woude CJ, Florencia EF, Prens EP. Hidradenitis suppurativa and inflammatory bowel disease: are they associated? Results of a pilot study. Br J Dermatol 2010;162:195-7.

7 Kimball AB, Jemec GB, Yang M et al. Assessing the validity, responsiveness and meaningfulness of the hidradenitis suppurativa clinical response (HiSCR) as the clinical endpoint for hidradenitis suppurativa treatment. Br J Dermatol 2014;171:1434-42.

8 Finlay AY, Khan GK. Dermatology Life Quality Index (DLQI) - a simple practical measure for routine clinical use. Clin Exp Dermatol 1994;19:210-6.

9 Wang B, Yang W, Wen W et al. Gamma-secretase gene mutations in familial acne inversa. Science 2010;330:1065.

10 Ingram JR, Wood M, John B, Butler R, Anstey AV. Absence of pathogenic $\gamma$-secretase mutations in a South Wales cohort of familial and sporadic hidradenitis suppurativa (acne inversa). Br J Dermatol 2013;168:874-6. 
11 von Laffert M, Stadie V, Wohlrab J, Marsch WC. Hidradenitis suppurativa/acne inversa: bilocated epithelial hyperplasia with very different sequelae. Br J Dermatol 2011;164:367-71.

12 Zouboulis CC, Desai N, Emtestam L et al. European S1 guideline for the treatment of hidradenitis suppurativa/acne inversa. J Eur Acad Dermatol Venereol 2015;29:619-44.

13 Ingram JR, McPhee M. Management of hidradenitis suppurativa: a UK survey of current practice. Br J Dermatol 2015, epub ahead of print.

14 Gener G, Canoui-Poitrine F, Revuz JE et al. Combination therapy with clindamycin and rifampicin for hidradenitis suppurativa: a series of 116 consecutive patients. Dermatology 2009;219:148-54.

15 Grant A, Gonzalez T, Montgomery MO, Cardenas V, Kerdel FA. Infliximab therapy for patients with moderate to severe hidradenitis suppurativa: a randomized, doubleblind, placebo-controlled crossover trial. J Am Acad Dermatol 2010;62:205-17.

16 Kimball AB, Kerdel F, Adams D et al. Adalimumab for the treatment of moderate to severe Hidradenitis suppurativa: a parallel randomized trial. Ann Intern Med 2012;157:846-55.
17 van der Zee HH, Prens EP, Boer J. Deroofing: a tissue-saving surgical technique for the treatment of mild to moderate hidradenitis suppurativa lesions. J Am Acad Dermatol 2010;63:475-80.

18 Buimer MG, Ankersmit MF, Wobbes T, Klinkenbijl JH. Surgical treatment of hidradenitis suppurativa with gentamicin sulfate: a prospective randomized study. Dermatol Surg 2008;34:224-7.

19 Ingram JR, Abbott R, Ghazavi $\mathrm{M}$ et al. The hidradenitis suppurativa priority setting partnership. Br J Dermatol 2014;171:1422-7.

20 von der Werth JM, Williams HC. The natural history of hidradenitis suppurativa. J Eur Acad Dermatol Venereol 2000;14:389-92.

Address for correspondence: Dr J Ingram, 3rd floor, Glamorgan House, University Hospital of Wales, Heath Park, Cardiff CF14 4XW, UK.

Email: ingramjr@cardiff.ac.uk 